\title{
Conditions and Consequences of Maintenance Rehearsal
}

\author{
John T. Wixted \\ University of California, San Diego
}

\begin{abstract}
The present experiments investigated the idea that the anticipated delay to initial recall governs whether a subject will rely on elaborative or maintenance rehearsal. In agreement with earlier research, the results suggested that maintenance rehearsal is used only when words can be rehearsed up to the moment of recall. A novel finding concerned the accessibility of the items processed in that way. When subjects were explicitly asked to recall those items first in final free recall, they were as likely to be recalled as items that had been processed elaboratively. This finding suggests that even rote rehearsal creates an accessible memory trace, albeit one that is most efficiently accessed by contextual rather than semantic cues.
\end{abstract}

Following the introduction of the levels of processing framework, one prominent line of research investigated the effects of various orienting tasks on delayed memory performance (Craik \& Lockhart, 1972; Craik \& Tulving, 1975). The typical finding from this literature was that tasks involving semantic analysis lead to better recall of words than tasks involving structural or phonemic analyses (Lewandowsky \& Hockley, 1987). A second line of research fostered by the levels of processing framework investigated the conditions under which subjects will naturally rely on different levels of processing when they are attempting to memorize a list of words. The central question addressed in this literature is whether subjects choose to devote semantic (or elaborative) rehearsal to some words and simple rote (or maintenance) rehearsal to others. Indeed, as described below, dynamic shifts in rehearsal strategy appear to be the norm. The experiments described herein were intended to further advance this line of inquiry, with particular emphasis on the conditions giving rise to and the enduring consequences of maintenance rehearsal.

Most of the research on the issue of rehearsal strategy is consistent with the idea that subjects use some form of elaborative rehearsal unless it is clear that the items being rehearsed can be maintained indefinitely (i.e., up to the moment of recall). When that condition obtains, elaborative strategies are essentially superfluous and may be abandoned in favor of simple maintenance rehearsal (Gotz \& Jacoby, 1974; Jacoby \& Bartz, 1972; Meunier, Ritz, \& Meunier, 1972; Roenker, 1974; Watkins \& Watkins, 1974). In a standard list memory procedure, the initial items of a list cannot be rehearsed indefinitely because of the incoming stream of subsequent items. Thus, the initial items would seem to be the most likely candidates for elaborative rehearsal. The last few items of a list, on the other hand, can be rehearsed unencumbered until the recall period begins just a few moments later. As a result, these items would seem to be the most likely candidates for

I would like to express thanks to Heidi Squire, Julie Baroway, Paul Havig, and Barbara Schneider for their assistance in data collection, and Robert Greene, Mark McDaniel, and Vito Modigliani for their thoughtful commentaries on this work.

Correspondence concerning this article should be addressed to John T. Wixted, Department of Psychology, C-009, University of California, San Diego, La Jolla, California 92093. maintenance rehearsal. In agreement with this analysis, Craik (1970) found that the serial position curve of final free recall (FFR) is characterized by a robust primacy effect and a negative recency effect (i.e., the last few items of each list were the least likely to be recalled). While not definitive, these results are certainly consistent with the idea that subjects shifted from elaborative rehearsal of the initial items of each list to maintenance rehearsal for the terminal items.

If subjects do employ rote rehearsal for items at the end of a list, then allowing extra rehearsal of those items should have no effect on FFR performance. Craik and Watkins (1973) tested this prediction and found that allowing $20 \mathrm{~s}$ of uninterrupted rehearsal for the last four words of a 12-item list had no effect on final recall. Wixted and McDowell (1989) extended this analysis by demonstrating that the effectiveness of rehearsal declines monotonically as a function of a word's serial position. More specifically, they found that allowing extra rehearsal of the initial items of a list greatly enhanced their probability of final recall, whereas extra rehearsal of the middle items had an intermediate effect, and (in agreement with Craik and Watkins) extra rehearsal of the last few items had no effect whatsoever. Thus, it seems that as subjects realize the end of the list is approaching, they rely increasingly on maintenance rehearsal (cf. Watkins \& Watkins, 1974).

On the other hand, Dalezman (1976) reported an interesting phenomenon that warrants some degree of caution in interpreting the findings reviewed above. In a final recall test, subjects in this experiment were asked to concentrate on recalling the last few items from each of the preceding lists before attempting to recall the remaining items. If the latter items of each list were actually processed by maintenance rehearsal, one might expect them to be unavailable in final recall regardless of output strategy. In contrast to this expectation, Dalezman found that the reverse output strategy resulted in a positive recency effect in FFR and a reduced (but still positive) primacy effect. Other groups of subjects who were given free-recall instructions, or who were asked to begin final recall with the initial items of each list, exhibited the usual negative recency effect. On the basis of these results, Dalezman argued that many findings taken to be indicative of differences occurring at input may actually be due to processes taking place at output. Thus, for example, the negative recency effect of FFR may arise in part because 
subjects usually attempt to recall the experimental lists in forward order. The successful implementation of this strategy would selectively disadvantage the last items of each list because of output interference (Roediger, 1974, 1978).

The present series of experiments was designed to pursue these matters in greater detail, beginning with a detailed analysis of the effect on rehearsal of anticipated delay to initial recall. The basic strategy involved varying the initial delay to recall over three levels for both rehearsed and nonrehearsed words. In the first experiment the delays were predictable in length, and in the second they were not. If anticipated delay to initial recall is the critical determinant of rehearsal strategy, it should be possible to occasion robust effects on FFR performance by simply cuing subjects in advance as to the size of the filled delay in effect on any given trial. The longer the cued delay, the more effective rehearsal should be. The same effects should not be observed when the delays to initial recall cannot be predicted in advance or when words are presented too rapidly to be rehearsed.

Once the conditions giving rise to differences in rehearsal in the present context were clearly established, the final experiment investigated the accessibility of those items that (on the basis of the first two experiments) were clearly processed by maintenance rehearsal. This experiment constitutes an essential attempt to replicate the surprising results reported by Dalezman (1976) and was intended to shed additional light on the accessibility of items processed without apparent elaboration. Surprisingly, when subjects were asked to attempt recall of those items first in FFR, they were as likely to be recalled as items that were presumably rehearsed elaboratively. In contrast to earlier research, however, the successful retrieval of these items did not correspondingly interfere with the retrieval of other items.

\section{Experiment 1}

Jacoby and Bartz (1972) and Roenker (1974) reported that the introduction of a filled delay before initial recall eliminated the negative recency effect of FFR. The same result was obtained when the potentially confounding effect of initial recall was eliminated by canceling initial recall for some items (Gotz \& Jacoby, 1974). The present experiment extends these analyses of precuing in two ways-first, by using three anticipated delays to evaluate the possibility of continuously changing rehearsal strategies and, second, by including a control condition in which rehearsal was minimized. As indicated above, the effect of precuing should be restricted to those items that have been rehearsed if the principal effect of that variable is to influence rehearsal strategy. If the effect of precuing remains evident for words that have not been rehearsed, then an explanation based on differences in rehearsal strategy might need to be abandoned in favor of an explanation based on differences in initial encoding.

The inclusion of a condition in which rehearsal is minimized also provides a baseline against which to gauge the effects of rehearsal. The conditions favoring maintenance rehearsal exist when subjects believe that the items being rehearsed can be easily maintained until the recall period begins. One may predict, but not require, that such rehearsal will have little effect on delayed recall performance relative to the no-rehearsal condition (cf. Glenberg \& Adams, 1978; Nelson, 1977). The conditions favoring various degrees of elaborative rehearsal exist when subjects believe that a filled delay will be interposed between rehearsal and recall. In this case, one may predict that such rehearsal will result in a higher level of delayed recall relative to the no-rehearsal condition.

The first experiment employed a multiple list-length procedure in which subjects were always cued as to the list length in effect. Lists of 5,10 , or 15 items were presented either very rapidly (allowing little time for rehearsal) or slowly enough to allow $15 \mathrm{~s}$ of overt rehearsal for each set of five items in the list. Of primary interest was the probability of final recall for the first five items of each list. For these words, the anticipated delay to initial recall increases as a function of list length. Thus, one might expect rehearsal of words in the 5-item lists to be relatively ineffective because they can easily be rehearsed up to the moment of recall (the conditions favoring maintenance rehearsal). Rehearsal of the first five words of the 10 item lists should be more effective and rehearsal of the corresponding words of the 15 -item lists still more effective because of their longer anticipated delays to initial recall.

The present design offers two important advantages over the use of a standard single-trial free-recall procedure in which words are presented sequentially at a constant rate. First, in the standard procedure, more time is available to rehearse the earlier items in the list compared with the later items (Rundus, 1971). The present procedure allows $15 \mathrm{~s}$ of unimpeded rehearsal for the first five words of each list, followed by an especially demanding distractor task (viz., the overt rehearsal of the next five words). Thus, the quantity of rehearsal for the items of interest, the first five words of each list, is essentially equated. Second, Wixted and McDowell (1989) speculated that the decrease in rehearsal efficacy as a function of proximity to recall might be attributed to increasing within-list proactive interference for the later items in a list. The present design circumvents this procedural confound because the focus is always on the first five words in each list.

\section{Method}

Subjects. The subjects were 22 undergraduates of the University of California, San Diego, who were enrolled in an introductory psychology course. Participation in the experiment satisfied a course requirement.

Materials and design. Lists of 5, 10, and 15 words were constructed from a pool of 540 high-frequency nouns drawn from Thorndike and Lorge (1944). For each subject, the lists were constructed by randomly selecting individual words from the word pool (a different random order of words was used for every subject). Three lists ( 1 of each size) served as practice lists and 12 lists ( 4 of each size) were used on experimental trials. As described below, half of the experimental lists were presented in a way that allowed ample time for rehearsal, and half were presented very rapidly to minimize rehearsal. The design of the experiment was completely within subjects-all subjects received all list sizes under the two rehearsal conditions. The order of list presentation and the rehearsal condition assigned to each list was determined randomly on line with the 
restriction that each list size occur twice under the two rehearsal conditions.

The lists were presented visually on a computer monitor, and subjects were instructed to read each word aloud as it was displayed. The words were always presented in sets of five in the following way. The first word of a set was presented just above the center of the screen. One-half s later, the second word was presented just beneath the first word. One-half $s$ after that, the third word was presented just beneath the second word, and so on until all five words were on the screen. One-half s later, all five words were removed from the screen.

For those lists that did not allow time for rehearsal, the presentation of the first set of five words was followed immediately by the recall period (for 5-item lists) or by the presentation of the next set of five words (for 10- and 15-item lists). Each set of five words was presented in the same way as the first. For lists that did allow time for rehearsal, the computer paused for $15 \mathrm{~s}$ after each set of five words was presented. More specifically, the letters of the five words were obscured with asterisks $1 / 2 \mathrm{~s}$ after the fifth word appeared on the screen. The asterisks remained on the screen for $15 \mathrm{~s}$, while the subjects overtly rehearsed the most recently presented set of words. After each new set was presented, the words were again obscured by asterisks for $15 \mathrm{~s}$, during which time the subjects overtly rehearsed them.

Once all of the words in the list were presented, the onset of recall was signaled by a series of question marks that appeared on the center of the screen. Subjects were allowed $90 \mathrm{~s}$ for written free recall.

Procedure. All subjects were tested individually. After signing a consent form, subjects were informed that they would be viewing lists of words on the screen that they would be asked to recall. They were further instructed to read the words aloud and, where appropriate, to rehearse aloud. The performance of all subjects was monitored by a research assistant to ensure continuous, overt rehearsal of the appropriate set of five words. Prior to the experimental trials, subjects received three practice trials involving lists of 5,10 , and 15 items. Two of these were randomly assigned to the rehearsal condition and one to the nonrehearsal condition.

Before each list was presented, the word ready appeared on the center of the screen, along with a number in parentheses. That number indicated to the subject the size of the upcoming list $(5,10$, or 15 words). The subjects were not informed about whether the list would allow time for rehearsal.

After the 3 practice and 12 experimental lists were completed, subjects were interviewed about rehearsal strategies they employed and debriefed about the purpose of the experiment. Following this (which required 3-4 min), subjects were asked to recall all of the items they had seen earlier in the session in any order they wished. The final recall period lasted for $10 \mathrm{~min}$, although subjects were allowed to leave before this if they insisted that they could recall no more words.

\section{Results and Discussion}

Subjects described a variety of rehearsal strategies, including rote repetition, creating sentences or stories, associating pairs of words, and imagery. In general, however, subjects reported that they used the same rehearsal strategy regardless of which words they were rehearsing. Of the 22 subjects who completed the experiment, 18 reported no conscious changes in rehearsal strategy, 2 reported that they concentrated especially hard on the initial items of the longer lists (because those words would be the most difficult to recall after the list was presented), and 2 reported that they concentrated especially hard on the last items of each list. The rationale for this strategy was that the initial items were always too difficult to recall and thus were not worth any effort to memorize them.

Of primary interest in this experiment is the probability of recall for the first five words of each list. As described earlier, subjects should adopt more effective rehearsal strategies for the first five words when the lists are known to involve 10 or 15 items then when they are known to involve only 5 items (self-reports to the contrary notwithstanding). Figure 1 shows the probability of initial and final recall (upper and lower panels, respectively) for the first five words of each list. As might be expected, the probability of initial recall falls off with increasing delay. Thus, the words in the 5-item lists were almost perfectly recalled initially because those words were either just presented or were still undergoing active rehearsal when the recall signal was presented. By contrast, recall of the first five words in the 10- and 15-item lists was delayed while additional sets of five words were presented and (in some cases) rehearsed. The apparent decline in probability of initial recall as a function of list length was confirmed by linear trend analyses, $F(1,21)=114.77, M S_{\mathrm{e}}=1.50$, and $F(1,21)$

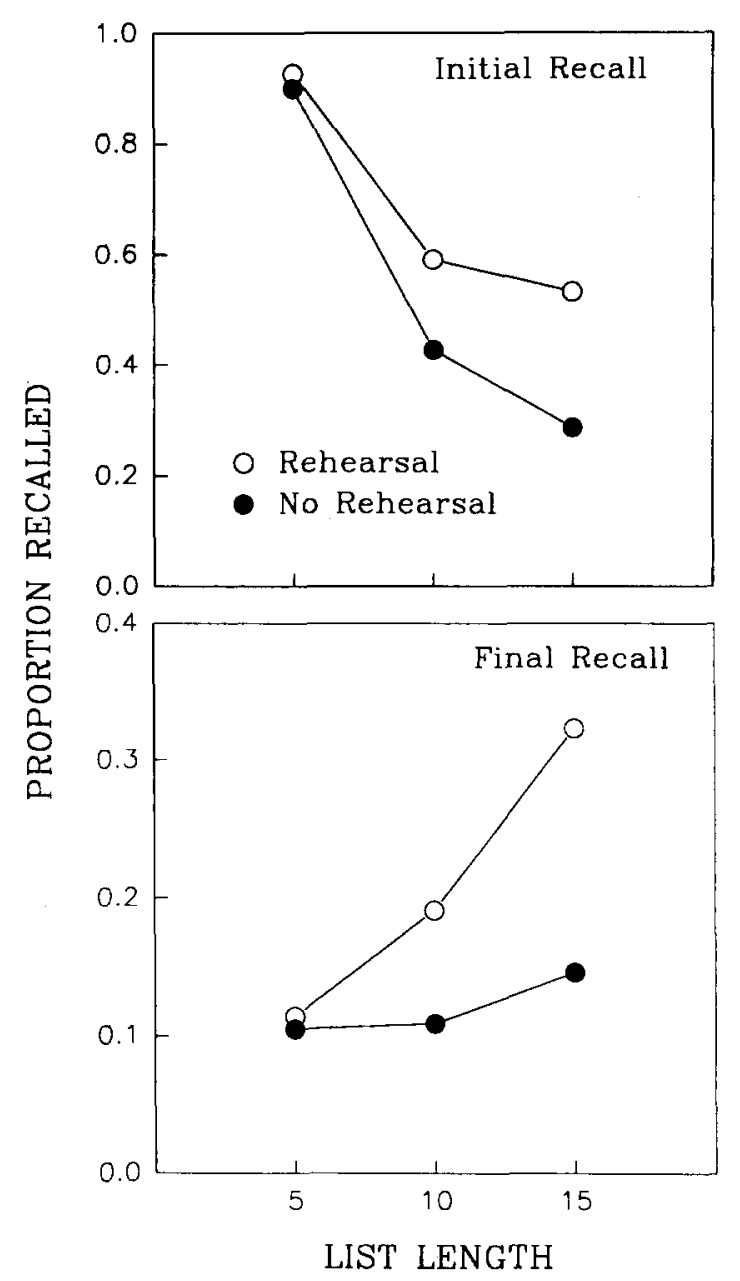

Figure 1. Probability of initial and final recall (upper and lower panels, respectively) of the first five words from lists of 5,10 , and 15 items in Experiment 1. 
$=160.2, M S_{\mathrm{e}}=2.59$ for the rehearsed and nonrehearsed words, respectively (unless noted otherwise, an $\alpha$ level of .05 was adopted for all statistical tests). An analysis of variance (ANOVA) performed on the initial recall data revealed main effects for rehearsal, $F(1,21)=21.5, M S_{\mathrm{e}}=3.25$, and list length, $F(2,42)=113.11, M S_{\mathrm{e}}=2.78$, as well as a significant interaction owing to the differing slopes of the two recall functions, $F(2,42)=5.04, M S_{\mathrm{e}}=2.65$.

The lower panel of Figure 1 shows that rehearsal of the first five words from the 5-item lists did nothing to enhance the final recall of those items compared with the no-rehearsal condition. By contrast, rehearsal of the first five words from the 10-item lists, and to an even greater extent the 15-item lists, clearly improved final recall. The apparent increase in probability of final recall as a function of list length for the rehearsed items was confirmed by a significant increasing linear trend, $F(1,21)=14.24, M S_{\mathrm{e}}=0.38$. By contrast, a trend analysis performed on the nonrehearsed items did not approach significance, $F(1,21)=1.35, M S_{\mathrm{e}}=1.365, p>.25$. The power of the latter test to correctly reject the null hypothesis is 0.88 , assuming a true difference of 0.10 between the endpoints of the no-rehearsal function. An ANOVA performed on the final recall data revealed main effects for rehearsal, $F(1,21)=9.75, M S_{\mathrm{e}}=2.71$, and list length, $F(2$, $42)=6.71, M S_{\mathrm{e}}=2.67$, as well as a significant interaction owing to the differing slopes of the two recall functions, $F(2$, $42)=3.79, M S_{\mathrm{e}}=2.06$.

The data considered above are clearly consistent with the notion that subjects adjust rehearsal strategies depending upon the anticipated delay to initial recall (cf. Gotz \& Jacoby, 1974). When subjects are aware that the words can be rehearsed indefinitely, rehearsal does not affect the probability of final recall. When they are aware that an intervening delay will occur, increasingly effective strategies are employed depending on the anticipated size of that delay. Although these findings would seem to imply a continuous adjustment in the kind of rehearsal employed, they do not necessarily rule out a continuous increase in the probability of switching from maintenance to elaborative rehearsal. Thus, for example, as the anticipated delay to initial recall increases, subjects may become more and more likely to employ an elaborative strategy and less and less likely to employ maintenance rehearsal. In either case, the results suggest that more effective strategies are employed with increasing anticipated delay to initial recall.

Several possible alternative explanations can be dismissed on the basis of these results. For example, the data are not consistent with the notion that a buildup of proactive interference might account for the decline in rehearsal effectiveness as subjects approach the end of a list (cf. Wixted \& McDowell, 1989). In this experiment, the words in question were always the first five words of the list. If anything, the first five words of the longer lists were disadvantaged by a higher level of within-list retroactive interference. Moreover, the results shown in Figure 1 weigh against the possibility that the effects of anticipated delay occur at the moment of initial encoding. If subjects allocated increased attention to the initial items of a long list, for example, one might expect to see some consequence of this in an increasing linear trend for the nonrehearsed items. No such trend was observed.
The present results that so clearly imply shifts from maintenance to elaborative rehearsal stand in curious contrast to the subjects' self-reports of unchanging rehearsal strategies. Although self-report data should be viewed with considerable skepticism (e.g., the data may primarily reflect the way in which the questions were worded), the finding is interesting and warrants further attention. Especially compelling support for the notion that subjects altered rehearsal strategy as a function of list length would be provided by spontaneous selfreport descriptions to that effect. Until such a result is obtained, however, it should be noted that these self-report data provide the only piece of evidence that is not fully consistent with the changing rehearsal account.

A potential interpretive difficulty arises whenever some words are associated with a longer delay to initial recall than other words. Specifically, some evidence suggests that the longer the actual delay over which a word is initially recalled, the more likely that word is to be recalled again in FFR (Modigliani, 1976, 1980; Modigliani \& Hedges, 1987). Thus, perhaps the reason why the initial words of the 15 -item lists were so easily retrieved in FFR was due to the fact that they were successfully retrieved over a relatively long delay in initial recall. Some evidence against this interpretation is apparent in Figure 1. Specifically, the nonrehearsed words did not exhibit an increasing trend in spite of the fact that the first five words of the 15-item lists were recalled initially over a longer delay than the corresponding words in the 10- and 5 -item lists. On the other hand, the magnitude of the initial delays for the lists that did not receive rehearsal were relatively short. Thus, for example, the delay to initial recall for the first five words of a 15-item list was only $5 \mathrm{~s}$ (the time required to present the remaining two sets of five words). Perhaps these initial delays were too short to differentially strengthen the initial items. The second experiment was designed to evaluate the possibility that the effects observed in Experiment 1 resulted from the strengthening effects of delayed initial recall for the rehearsed items.

\section{Experiment 2}

The design of this experiment was identical to that of the first experiment except that subjects were never informed in advance of the length of the list. Because subjects could not determine the size of the list while rehearsing the first five words, this procedure effectively equates the type of rehearsal applied to those words. On the other hand, the delay over which the initial items must be recalled still increases substantially with list length. If delayed recall differentially strengthens the initial items of the 10- and 15-item lists, then the same pattern of results that were observed in Experiment 1 should be obtained again. By contrast, if the type of rehearsal is the primary determinant of FFR performance, then a flat recall function should be obtained for both the rehearsed and the nonrehearsed words.

\section{Method}

Subjects. The subjects were 24 undergraduates of the University of California, San Diego, who were enrolled in an introductory 
psychology course. Participation in the experiment satisfied a course requirement.

Materials and design. Lists of 5, 10, and 15 words were again constructed from a pool of 540 high-frequency nouns drawn from Thorndike and Lorge (1944). Different lists were constructed for each subject by selecting words randomly from the word pool. As before, half of the lists were presented in a way that allowed ample time for rehearsal, and half were presented very rapidly to minimize rehearsal.

Procedure. The procedure was identical to that used in Experiment 1 with one exception. Before each list was presented, the word ready appeared on the center of the screen without any additional indication of the size of the impending list.

\section{Results and Discussion}

As before, the analyses described below focus on the probability of recall for the first five words of each list. Figure 2 shows the probability of initial and final recall (upper and lower panels, respectively) for the first five words of each list. Again, as expected, the probability of initial recall falls off with list length. Thus, the words in the 5-item lists were very likely to be recalled initially because those words were either

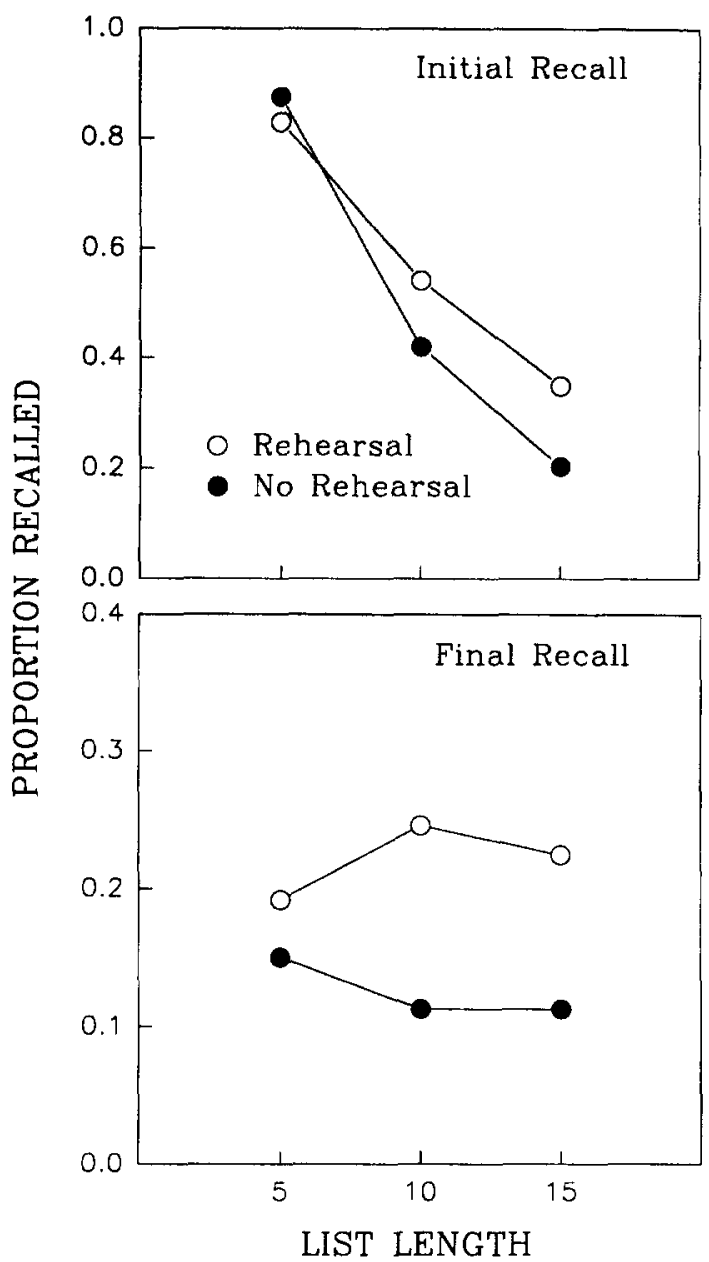

Figure 2. Probability of initial and final recall (upper and lower panels, respectively) of the first five words from lists of 5,10 , and 15 items in Experiment 2. just presented or were still undergoing active rehearsal when the recall signal was presented. By contrast, recall of the first five words in the 10-item and 15-item lists was delayed while additional sets of words were presented and (for some of the lists) rehearsed. Once again, the apparent decline in initial recall as a function of list length was confirmed by highly significant decreasing linear trends, $F(1,23)=105.65, M S_{\mathrm{e}}=$ 2.61 and $F(1,23)=421.33, M S_{\mathrm{e}}=1.28$ for the rehearsed and nonrehearsed words, respectively. An ANOVA performed on the initial recall data revealed a main effect for rehearsal, $F(1$, 23) $=9.76, M S_{\mathrm{e}}=2.00$, a main effect for list length, $F(2,46)$ $=135.03, M S_{\mathrm{e}}=3.02$, and a significant interaction owing to the differing slopes of the two function, $F(2,42)=6.66, M S_{c}$ $=1.96$.

Although the probability of initial recall declines with list length, the lower panel of Figure 2 reveals that the final recall functions for both the rehearsed and nonrehearsed words is essentially flat. A trend analysis performed on the data from the rehearsal condition did not approach significance, $F(1$, 23) $=0.33, M S_{\mathrm{e}}=4.029, p>.50$. The power of this test to correctly reject the null hypothesis is .53 , assuming a true difference of 0.10 between the endpoints of the rehearsal function. This finding contrasts with the result shown in Figure 1 and suggests that differences in delay to initial recall do not account for the increase in final recall probability observed in the previous experiment. As with the rehearsed items, a trend analysis performed on the data from the norehearsal condition did not approach significance, $F(1,23)=$ $1.302, M S_{\mathrm{e}}=1.30, p>.25$. The power of this test to correctly reject the null hypothesis is .92 , assuming a true difference of 0.10 between the endpoints of the no-rehearsal function. An ANOVA performed on the final recall data revealed a main effect for rehearsal (the rehearsed items were more likely to be recalled than the nonrehearsed items), $F(1,23)=18.65$, $M S_{\mathrm{e}}=1.77$, no effect of list length and no interaction. On the whole, the results of this experiment would appear to weigh against a delayed recall interpretation of the results of Experiment 1.

In some respects the present results may seem somewhat surprising. Previous research has demonstrated that an initial recall test enhances the probability of final recall (Darley \& Murdock, 1971) and, more specifically, that delayed initial recall exerts a greater strengthening effect on FFR performance than immediate (or nondelayed) initial recall (Modigliani, 1976, 1980). The present results suggest that differential strengthening of this kind, if it occurred, was not sufficient to account for the increasing FFR trend observed in Experiment 1. On the other hand, this conclusion, which is the central conclusion of the present experiment, does not necessarily imply an absence of differential strengthening. Conceivably, the few words initially recalled from the 15 -item lists were strengthened to a greater extent than the many words initially recalled from the 5 -item lists. The net result might be an FFR function that is essentially flat, such as that observed in Experiment 2. In any case, whether or not words were differentially strengthened during initial recall, the increasing trend observed in Experiment 1 was apparently an independent phenomenon.

The results of Experiment 2 also serve to rule out an unlikely explanation of Experiment 1 based on surreptitious 
rehearsal. Although the distractor task in effect after the first five words were presented seems even more demanding than standard distractor tasks (such as counting backwards by threes), it could be argued that subjects nevertheless managed to devote extra rehearsal to the initial set of five words in the longer lists (thus accounting for the increase in probability of recall as a function of list length). An account based on the notion of surreptitious rehearsal incorrectly predicts the same effect in Experiment 2.

At first glance, the final recall data depicted in Figure 2 appear to be at odds with the well-known list-length effect, which states that items on a longer list are remembered less well than items on a shorter list (e.g., Ratcliff, Clark, \& Shiffrin, 1990). Whereas this effect certainly holds for initial recall, it is not at all apparent in the final recall data shown in Figure 2. A theoretical account for the list-length effect offered by Gillund and Shiffrin (1984) basically holds that individual word representations, or images, are sampled until a stopping criterion is satisfied. Because the same stopping criterion is used regardless of list size, individual items are less likely to be sampled after a long list than a short one. In final recall, however, subjects probably do not attempt to retrieve items on a list-by-list basis. Instead, all of the preceding words may be regarded as one large set. In that case, an account such as that offered by Gillund and Shiffrin (1984) would not predict a list-length effect because all of the items are equally likely to be sampled before the stopping criterion is satisfied.

\section{Experiment 3}

It seems clear from the first two experiments that when subjects are aware that words can be rehearsed up to the moment of recall, they do not employ effective nmemonic strategies and instead adopt a strategy of rote rehearsal. The purpose of the third experiment was to shed additional light on the memorial status of words rehearsed in that way. One possibility, originally advanced by Craik and Lockhart (1972), is that maintenance rehearsal produces no lasting effect whatsoever. According to this view, once these items are no longer undergoing active maintenance rehearsal, they could not be remembered under any circumstances. However, Glenberg, Smith, and Green (1977) and Glenberg and Adams (1978) found that although rote rehearsal does not facilitate recall, it does facilitate recognition (cf. Woodward, Bjork, \& Jongeward, 1973). In other words, although maintenance rehearsal does not create the associative pathways necessary to facilitate recall, it does affect some aspect of the memory trace that allows the rehearsed items to be discriminated from distractors on a recognition test. In particular, an analysis of error patterns performed by Glenberg and Adams (1978) suggested that maintenance rehearsal strengthens the acoustic or phonemic components of the memory trace.

On the basis of this analysis, one would not expect variations in retrieval strategy to have much of an effect on the recallability of items processed by rote rehearsal. However, as indicated earlier, Dalezman (1976) reported that the last few items of a list (which are usually assumed to be processed by maintenance rehearsal) are in fact accessible if subjects are instructed to recall those items before attempting to recall the other items on a list. A finding such as this would either suggest that subjects did not rely on maintenance rehearsal after all or that such rehearsal creates a more accessible memory trace than might otherwise be expected. Dalezman emphasized the former view and suggested that differences in FFR performance taken to be indicative of different rehearsal strategies may actually be the result of differences in output strategy: items recalled first suppress or interfere with the recall of other items (Roediger, 1974, 1978).

On the other hand, Dalezman's results have never been replicated. Indeed, under some list presentation conditions, output interference effects appear to be minimal. Whitten (1978), for example, reported no significant effect of output order using a long-term paradigm (Bjork \& Whitten, 1974). In this experiment, subjects were presented with nine pairs of words, each preceded and followed by a 12-s distractor task (the last pair was followed by a 30-s distractor task). Whether subjects initiated recall with the beginning, middle, or terminal items, pronounced long-term serial position effects (both primacy and recency) were clearly evident.

In the following experiment, the accessibility of words thought to be processed by maintenance rehearsal (i.e., words in the predictable 5-item lists) was addressed. What would happen if subjects were unexpectedly cued in final recall to attempt retrieval of those items first? On the basis of the findings reported by Dalezman, one might expect an increase in the probability of recall of these words at the expense of the other words from the 10 - and 15 -item lists. On the other hand, if accessibility is fully established during list presentation (cf. Whitten, 1978), or the only effect of maintenance rehearsal is to strengthen the phomenic aspects of the memory trace (cf. Glenberg \& Adams, 1978), one would not expect this manipulation to appreciably alter the pattern of results depicted in Figure 1.

\section{Method}

Subjects. The subjects were 48 undergraduates of the University of California, San Diego who were enrolled in an introductory psychology course. Participation in the experiment satisfied a course requirement.

Materials and design. List construction and manner of list presentation were identical to those of Experiment 1 (i.e., all lists were predictable in length). List size $(5,10$, or 15 items) and rehearsal condition (rehearsed or not rehearsed) were varied within subjects, whereas FFR strategy (free vs. 5-item lists first) was varied between subjects. Subjects were randomly assigned to output condition with 23 subjects participating in the 5-first condition and 25 participating in the free condition (the imbalance was due to a scheduling error).

Procedure. The initial recall procedure was in all respects identical to Experiment 1. After the 3 practice and 12 experimental lists were completed, subjects were interviewed about rehearsal strategies they employed and debriefed about the purpose of the experiment. After this (which required 3 to $4 \mathrm{~min}$ ), subjects were asked to recall all of the items they had seen earlier in the session. Half the subjects were asked to recall the items in any order they wished (as in Experiment 1). The other half were asked to attempt recall of the items that had appeared in five-item lists only. After approximately 5 min engaged in this activity, these subjects were instructed to recall any of the 
remaining items they could remember. The final recall period lasted for $10 \mathrm{~min}$ for both groups.

\section{Results and Discussion}

Because the free-recall condition of Experiment 3 constitutes a replication of Experiment 1, the results from that condition will be considered first. Figure 3 shows the probability of initial and final recall from this condition for the first five words of each list. Once again, the rapid decline in the initial recall of the first five words as a function of list length was confirmed by highly significant linear trends, $F(1,24)=$ 119.91, $M S_{\mathrm{e}}=2.99$, and $F(1,24)=278.19, M S_{\mathrm{e}}=2.05$, for the rehearsed and nonrehearsed words, respectively. An ANOVA performed on the initial recall data revealed main effects for rehearsal and list length, $F(1,24)=14.43, M S_{\mathrm{e}}=$ 2.33 , and $F(2,48)=166.78, M S_{e}=3.07$, respectively, as well as a significant interaction, $F(2,48)=3.60, M S_{\varepsilon}=1.76$.

The lower panel of Figure 3 shows that, in final recall, rehearsal of words in the 5-item list did not increase their probability of final free recall, whereas rehearsal of the corre-

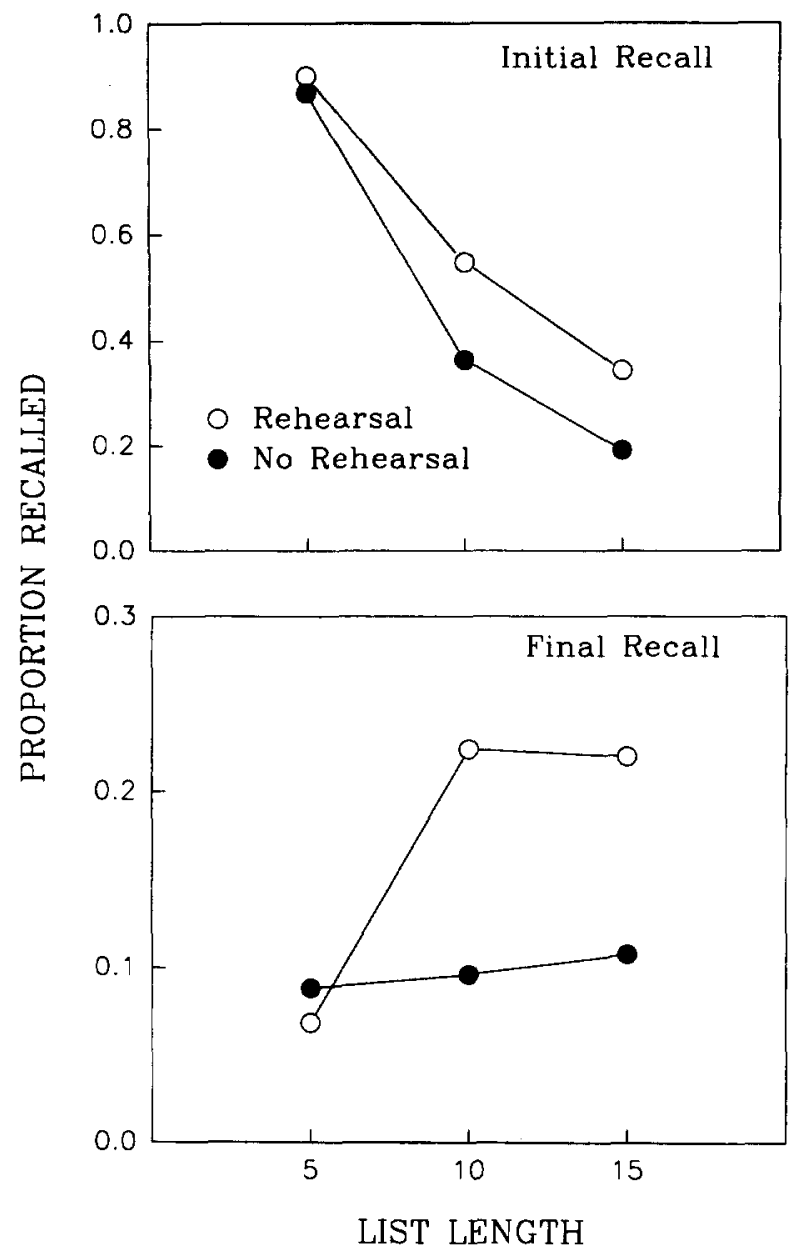

Figure 3. Probability of initial and final recall (upper and lower panels, respectively) of the first five words from the free condition in Experiment 3. sponding words in the 10- and 15-item lists did. These observations were confirmed by a significant, increasing linear trend for the rehearsed items, $F(1,24)=13.56, M S_{\mathrm{e}}=2.13$, and the absence of a significant trend for the nonrehearsed items. Further, a within-subjects ANOVA performed on the final recall data revealed main effects for rehearsal condition, $F(1,24)=7.30, M S_{\mathrm{e}}=2.76$, and list length, $F(2,48)=5.17$, $M S_{\mathrm{e}}=2.28$, as well as a significant interaction deriving from the different slopes of the two functions, $F(2,48)=3.65$, $M S_{\mathrm{e}}=2.26$.

These results essentially match those of Experiment 1 and suggest that subjects employ more effective rehearsal strategies when they anticipate a filled delay. One difference between these results and those of the first experiment is that the first five words of the 10 -item lists were as likely to be recalled as the corresponding words of the 15-item lists. Whereas the results from Experiment 1 and those reported by Wixted and McDowell (1989) imply a more continuous shift in rehearsal strategy as a function of anticipated delay, the results shown in Figure 3 suggest that a substantial delay of any size introduced before initial recall may induce the same level of elaborative rehearsal. The source of the discrepancy between this result and earlier findings is not immediately apparent.

Figure 4 shows the initial recall data from the free and 5 first conditions. The conditions of initial recall were identical for the two groups, and no differences between them would be expected. Indeed, the functions for the rehearsed words (upper panel) virtually overlap, and the same is true for the unrehearsed words (lower panel). An ANOVA performed on these data revealed no significant effects involving the condition factor.

The major question addressed by this experiment concerned the effect of output strategy on the accessibility of words from the 5-item lists. That is, was the probability of final recall for words in the 5-item lists increased when subjects were instructed to focus their recall efforts on those words first? A secondary issue concerned whether an output strategy giving priority to those words would interfere with final recall of words from the 10- and 15-item lists. Figure 5 compares final recall of words from the free and 5-first output conditions. The upper panel compares the recall functions for words that received rehearsal, and the lower panel compares the recall functions for words that did not. For the rehearsed words, the increasing trend apparent in the free condition is no longer apparent in the 5-first condition, $F(1,22)=0.25$, $M S_{\mathrm{e}}=3.19, p>0.50$. The power of this test to correctly reject the null hypothesis is .61, assuming a true difference of 0.10 between the endpoints of the function.

For the nonrehearsed words, the 5-first conditions exhibits a slight increase in the probability of recall for words from the 5-item lists and a slight decrease in the probability of recall for the words from the 15 -item lists. Indeed, while the no-rehearsal function from the free condition was flat (as in the previous experiments), the corresponding function from the 5-first exhibits a decreasing trend, $F(1,22)=6.84, M S_{\mathrm{e}}=$ 0.92 . Thus, the list-length effect that was not obtained in the preceding experiments for words assumed to be given equivalent processing was obtained here. This result is considered in more detail later. 


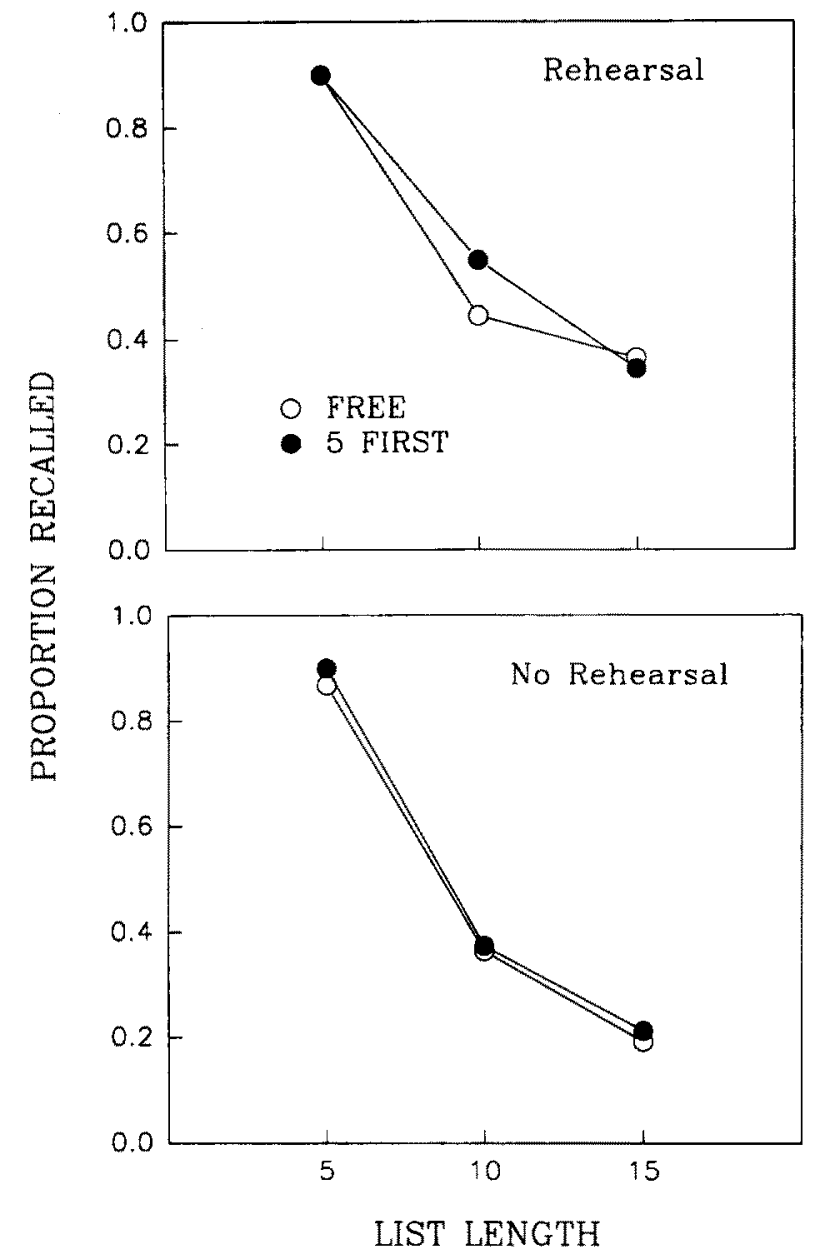

Figure 4. Probability of initial recall for the first five words from the free and 5-first conditions (upper and lower panels, respectively) in Experiment 3.

To further evaluate the effects of output strategy on recall, the data from the 5-item lists were analyzed separately. An ANOVA performed on these data revealed a significant effect of output condition (5-first exceeded free), $F(1,46)=9.01$, $M S_{\mathrm{c}}=1.83$, no effect of rehearsal, and a marginally significant interaction between output condition and rehearsal, $F(1,46)$ $=3.41, M S_{\mathrm{e}}=1.15, p=.07$. Planned follow-up analyses using the Bonferroni $t$ statistic served to clarify these effects.

For the rehearsed words (upper panel), instructing subjects to initiate output with items from the 5-item lists resulted in a substantial increase in final recall probability (from .07 to .19). This difference was highly significant, $t(1,46)=3.43$. For the nonrehearsed words (lower panel), instructing subjects to initiate recall with items from the 5-item lists also resulted in some improvement (from .09 to .13), but the difference between the two conditions did not approach significance, $t(1,46)=1.23, p>.20$. The power of this test to correctly reject the null hypothesis is 90 , assuming a true difference of 0.10 . Thus, although the data support the conclusion that output strategy influences accessibility of rehearsed words, the same cannot be said of the words that were not rehearsed.
An examination of the recall probabilities for the remaining sets of words from the 10- and 15-item lists failed to reveal any convincing evidence of output interference resulting from the 5-first output strategy. Table 1 presents the final probability of recall for each set of five words from the free and 5first output conditions. In most cases, the final recall probabilities from the 5-first condition equal or slightly exceed those from the free condition. The only exception involved the first five words from the 15 -item lists.

Although, on the whole, evidence for output interference is lacking, the no-rehearsal data from the 5-first condition do suggest some effect of output strategy. Specifically, the significantly decreasing linear trend suggests, for the first time in the present series of experiments, a list-length effect. As indicated earlier, an account of the list-length effect offered by Gillund and Shiffrin (1984) holds that subjects sample list items until a stopping criterion is satisfied. In final recall, if all of the preceding items are regarded as one large set, then one might not expect to find a list-length effect for equivalently processed items. On the other hand, if words from the five-item lists are searched first (as in the 5-first condition),

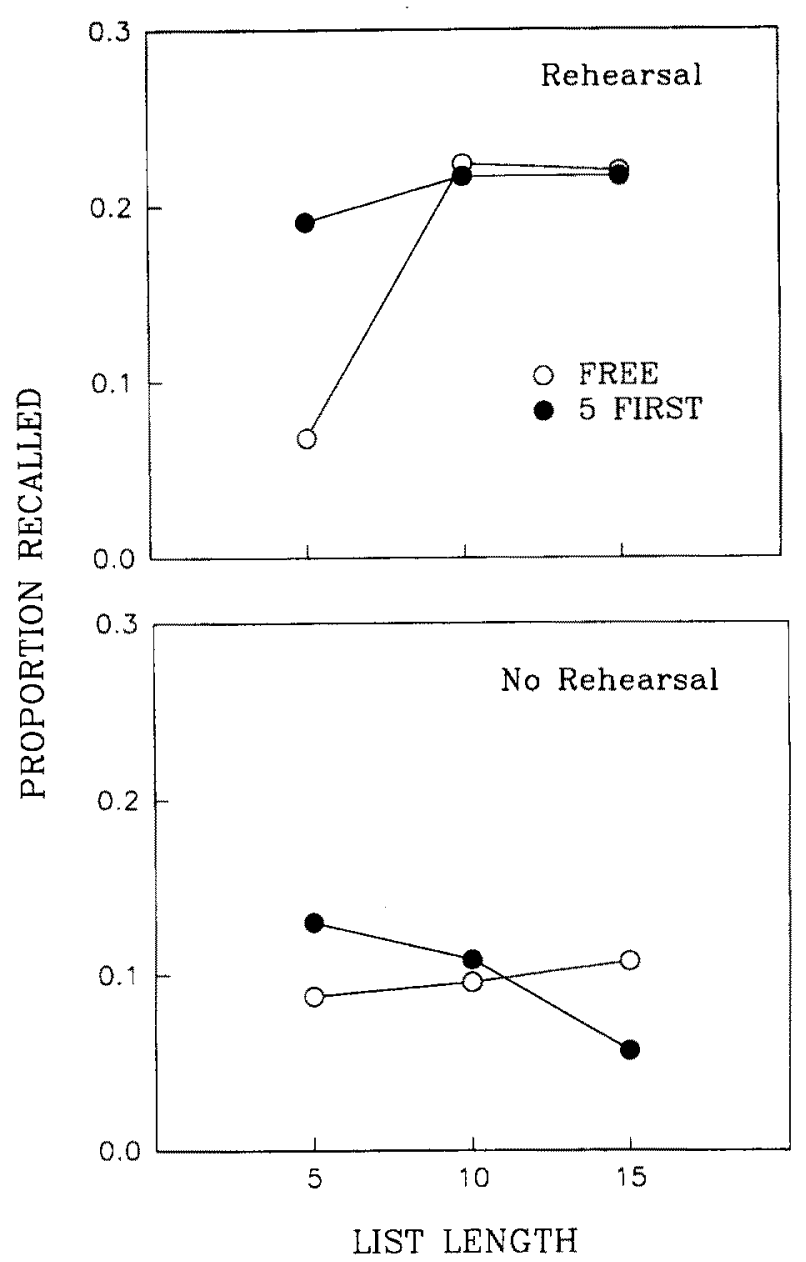

Figure 5. Probability of final recall for the first five words from the free and 5-first conditions (upper and lower panels, respectively) in Experiment 3. 
Table 1

Recall Probabilities for Individual Sets of Five Words

\begin{tabular}{|c|c|c|c|c|c|c|}
\hline \multirow[b]{2}{*}{ LL } & \multicolumn{2}{|c|}{1} & \multicolumn{2}{|c|}{2} & \multicolumn{2}{|c|}{3} \\
\hline & Free & 5 -first & Free & 5-first & Free & 5-first \\
\hline \multicolumn{7}{|c|}{ Rehearsed } \\
\hline 5 & 0.07 & 0.19 & - & - & - & - \\
\hline 10 & 0.22 & 0.22 & 0.13 & 0.15 & - & - \\
\hline 15 & 0.22 & 0.22 & 0.09 & 0.16 & 0.12 & 0.15 \\
\hline \multicolumn{7}{|c|}{ Unrehearsed } \\
\hline 5 & 0.09 & 0.13 & - & - & - & - \\
\hline 10 & 0.10 & 0.11 & 0.04 & 0.06 & - & - \\
\hline 15 & 0.11 & 0.06 & 0.09 & 0.10 & 0.07 & 0.10 \\
\hline
\end{tabular}

Note. Each pair of numbers represents values from the free and 5first conditions. $L L=$ list length and the column headings $(1,2$, and 3 ) denote the first, second, and third sets of five words in the list.

one might expect to find some evidence of the list-length effect in final recall after all. Indeed, such an effect is apparent in Figure 5. One might also expect to fine some evidence in the recall of other words as well, but Table 1 does not reveal any evidence of that kind. Thus, while Gillund and Shiffrin's model offers a reasonable interpretation of the apparent listlength effect obtained here, the present data are not entirely consistent with that account.

Although earlier studies have shown that prolonged maintenance rehearsal can facilitate recall (e.g., Rundus, 1980), the present results demonstrate that even under conditions in which maintenance rehearsal has apparently failed to facilitate recall, the items are accessible if they are recalled first. In that respect, these findings replicate those reported by Dalezman (1976). On the other hand, the successful retrieval of words from the 5-item lists did not occur at the expense of words thought to have received elaborative rehearsal (viz., those from the 10- and 15-item lists). This result is unique in this context and appears to warrant some modification in current theories about the effects of maintenance rehearsal.

\section{General Discussion}

The principal conclusions to be drawn from the present research are as follows. First, in agreement with earlier research, when subjects anticipate a long filled delay to initial recall, rehearsal tends to improve FFR performance. Second, the same result is not observed for items that are presented too rapidly to receive a significant amount of rehearsal. This comparison was necessary to rule out the possibility that anticipated delay exerted its effects on initial encoding rather than subsequent rehearsal. Third, the increasing probability of final recall as a function of anticipated delay to initial recall is not an artifact of actual delay to initial recall (which, as shown in Experiment 2, had very little effect on overall FFR performance). Apparently, subjects rehearse items more effectively as the anticipated delay increases (cf. Gotz \& Jacoby, 1974). Fourth, apparently inaccessible items (viz., those thought to be processed by maintenance rehearsal) are sur- prisingly accessible in FFR when subjects are instructed to focus their attention on recalling those items before attempting to recall other items. Contrary to earlier research, however, the successful recall of those items does not necessarily occur at the expense of other items not specifically cued for recall.

From a theoretical standpoint, the most interesting question raised by the present results concerns why seemingly inaccessible items (those assumed to have been accorded maintenance rehearsal) can be retrieved, given the appropriate output strategy. One potential explanation is that those words were erroneously judged to have been processed by maintenance rehearsal. In the absence of any apparent incentive, subjects may have nevertheless continued to employ elaborative strategies, even for words that could be rehearsed indefinitely. Weak support for this notion is provided by self-report data. With few exceptions, subjects reported that they did not change their conscious rehearsal strategies as a function of proximity to initial recall (cf. Shaughnessy, 1981).

The assumption that rehearsal strategies remained constant regardless of list size, however, is difficult to reconcile with the overall pattern of results. In line with the arguments advanced by Dalezman (1976), one might attempt to invoke output interference to explain the increasing probability of final recall as a function of anticipated delay to initial recall. That is, perhaps subjects chose to focus on retrieving items from the longer lists before attempting to retrieve items from the shorter lists, thereby placing the latter at a disadvantage. If so, why was the same effect not observed in Experiment 2, when list length was unpredictable? Even more damaging to this account is the lack of output interference effects in Experiment 3. Although instructing subjects to initiate recall with items from the shorter lists significantly increased their chances of recall, that effect did not occur at the expense of the other items from the longer lists (the only exception being the first five words from the 15-item lists in the no-rehearsal condition).

A closer analysis of the results reported by Dalezman (1976) reveals an outcome more similar than different to that reported here. In that experiment, requiring subjects to initiate final recall with the last few items of each list enhanced recall for those items and reduced the probability of recall for items at the beginning of each list. However, the reduction in recall probability for the primacy items was slight (from .28 to .26 for the first five items), whereas the increment in recall probability for the recency items was more substantial (from .19 to .25 for the last five items). Because the primacy effect was still robust and still slightly exceeded the magnitude of the positive recency effect produced by this procedure, the overall pattern of results do not point to an especially strong role for output interference. Some other factor appears to be responsible for the observed effect.

The results of the present research, as well as those of Dalezman upon further reflection, seem to suggest that subjects did indeed employ maintenance rehearsal when they realized that items could be rehearsed indefinitely and that such rehearsal has a more complex effect on memory than might be suggested by prevailing theories. As indicated earlier, the original levels of processing account suggested that maintenance rehearsal produces no lasting effect whatsoever (e.g., 
Craik \& Lockhart, 1972; Craik \& Watkins, 1973; Rundus, 1977), and a later modification suggested that it strengthens the phonemic components of the memory trace without creating the associative pathways that might facilitate delayed recall (e.g., Glenberg \& Adams, 1978). Baddeley (1990) recently proposed a similar account, according to which maintenance rehearsal serves to "prime" existing representations without creating associations between previously unrelated items or between an item and its experimental context. According to these theories, one would not expect a retrieval strategy of the sort used in Experiment 3 to significantly affect accessibility. In contrast to this expectation, the use of a salient contextual cue to guide recall (viz., list length) had a considerable impact on FFR performance.

As indicated earlier, Rundus (1980) demonstrated that prolonged maintenance rehearsal can increase the probability of delayed recall. In attempting to account for this result, he suggested that although rote, maintenance rehearsal does not serve to establish semantic associations, it may, by virtue of contiguity alone, establish an association between a word and some aspect of the experimental context. To the extent that these contextual cues are employed in the memory test, the mnemonic effects of such rehearsal will be revealed. This analysis appears to offer the most conservative interpretation of the present findings as well. Although subjects employed rote rehearsal when faced with five-item lists of known length, such rehearsal did more than prime the memory trace; it established an association between the rehearsed words and a salient feature of their context (viz., the fact that they appeared in a five-item list). On the subsequent memory test, instructing subjects to rely on that contextual feature facilitated access (cf. Glenberg, Bradley, Kraus, \& Renzaglia, 1983).

A more complex analysis of the present findings might appeal to a combined role for contextual retrieval and output interference. More specifically, an output interference effect specific to words accorded maintenance rehearsal remains a possibility. In Experiment 3, the contextual retrieval cue (namely, the instruction to recall words from the five-item lists) was presented before subjects attempted to recall the more well-rehearsed items from the longer lists. If the presentation of that cue were delayed, say, until the end of the FFR period, its effectiveness might have been diminished. Such an outcome would suggest that words processed by maintenance rehearsal are differentially susceptible to output interference. By contrast, words processed elaboratively appear to be relatively invulnerable to such interference (e.g., Table 1). Although this account does not explain the absence of an output interference effect for most of the nonrehearsed words, future research should investigate the possible interaction between different levels of processing and the sometimes elusive output interference phenomenon (cf. Roediger \& Schmidt, 1980).

\section{References}

Baddeley, A. (1990). Human memory: Theory and practice. Needham Heights, MA: Allyn and Bacon.

Bjork, R. A., \& Whitten, W. B. (1974). Recency-sensitive retrieval processes in long-term free recall. Cognitive Psychology, 6, 173189.

Craik, F. I. M. (1970). The fate of primary memory items in free recall. Journal of Verbal Learning and Verbal Behavior, 9, 143148.

Craik, F. I. M., \& Lockhart, R. S. (1972). Levels of processing: A framework for memory research. Journal of Verbal Learning and Verbal Behavior, 11, 671-684.

Craik, F. I. M., \& Tulving, E. (1975). Depth of processing and the retention of words in episodic memory. Journal of Experimental Psychology: General, 104, 268-294.

Craik, F. I. M., \& Watkins, M. J. (1973). The role of rehearsal in short-term memory. Journal of Verbal Learning and Verbal Behavior, 12, 599-607.

Dalezman, J. J. (1976). Effects of output on immediate, delayed, and final recall performance. Journal of Experimental Psychology: $\mathrm{Hu}$ man Learning and Memory, 2, 597-608.

Darley, C. F., \& Murdock, B. B. (1971). Effects of prior free recall testing on final recall and recognition. Journal of Experimental Psychology, 91, 66-73.

Gillund, G., \& Shiffrin, R. M. (1984). A retrieval model for both recognition and recall. Psychological Review, 91, 1-67.

Glenberg, A., \& Adams, F. (1978). Type I rehearsal and recognition. Journal of Verbal Learning and Verbal Behavior, 17, 455-463.

Glenberg, A. M., Bradley, M. M., Kraus, T. A., \& Renzaglia, G. J. (1983). Studies of the long-term recency effect: Support for a contextually guided retrieval hypothesis. Journal of Experimental Psychology: Learning, Memory, and Cognition, 9, 231-255.

Glenberg, A., Smith, S. M., \& Green, C. (1977). Type I rehearsal: Maintenance and more. Journal of Verbal Learning and Verbal Behavior, 16, 339-352.

Gotz, A., \& Jacoby, L. L. (1974). Encoding and retrieval processes in long-term retention. Journal of Experimental Psychology, 102, 291-297.

Jacoby, L. L., \& Bartz, W. H. (1972). Rehearsal and transfer to LTM. Journal of Verbal Learning and Verbal Behavior, 11, 561-565.

Lewandowsky, S., \& Hockley, W. E. (1987). Does CHARM need depth? Similarity and levels-of-processing effects in cued recall. Journal of Experimental Psychology: Learning, Memory, and Cognition, 13, 443-455.

Meunier, G. F., Ritz, D., \& Meunier, J. A. (1972). Rehearsal of individual items in short-term memory. Journal of Experimental Psychology, 95, 465-467.

Modigliani, V. (1976). Effects on a later recall by delaying initial recall. Journal of Experimental Psychology: Human Learning and Memory, 2, 609-622.

Modigliani, V. (1980). Immediate rehearsal and initial retention interval in free recall. Journal of Experimental Psychology: Human Learning and Memory, 6, 241-253.

Modigliani, V., \& Hedges, D. G. (1987). Distributed rehearsals and the primacy effect in single-trial free recall. Journal of Experimental Psychology: Learning, Memory, and Cognition, 13, 426-436.

Nelson, T. O. (1977). Repetition and depth of processing. Journal of Verbal Learning and Verbal Behavior, 16, 151-171.

Ratcliff, R., Clark, S. E., \& Shiffrin, R. M. (1990). List-strength effect: I. Data and discussion. Joumal of Experimental Psychology: Learning, Memory, and Cognition, 16, 163-178.

Roediger, H. L. (1974) Inhibiting effects of recall. Memory \& Cognition, 2, 261-269.

Roediger, H. L. (1978). Recall as a self-limiting process. Memory \& Cognition, 6, 54-63.

Roediger, H. L., \& Schmidt, S. R. (1980). Output interference in the recall of categorized and paired-associate lists. Journal of Experimental Psychology: Human Learning and Memory, 6, 91-105.

Roenker, D. L. (1974). Role of rehearsal in long-term retention. 
Journal of Experimental Psychology, 103, 368-371.

Rundus, D. (1971). Analysis of rehearsal processes in free recall. Journal of Experimental Psychology, 89, 63-77.

Rundus, D. (1977). Maintenance rehearsal and single-level processing. Journal of Verbal Learning and Verbal Behavior, 16, 665-681.

Rundus, D. (1980). Maintenance rehearsal and long-term recency. Memory \& Cognition, 8, 226-230.

Shaughnessy, J. J. (1981). Memory monitoring accuracy and modification of rehearsal strategies. Journal of Verbal Learning and Verbal Behavior, 20, 216-230.

Thorndike, E. L., \& Lorge, 1. (1944). The teacher's word book of 30,000 words. New York: Columbia University Press.

Watkins, M. J., \& Watkins, O. C. (1974). Processing of recency items for free recall. Journal of Experimental Psychology, 102, 488-493.
Whitten, W. B. (1978). Output interference and long-term serial position effects. Journal of Experimental Psychology: Human Learning and Memory, 4, 658-692.

Wixted, J. T., \& McDowell, J. J. (1989). Contributions to the functional analysis of single-trial free recall. Journal of Experimental Psychology: Learning, Memory, and Cognition, 15, 685-697.

Woodward, A. E., Bjork, R. A., \& Jongeward, R. H. (1973). Recall and recognition as a function of primary rehearsal. Journal of Verbal Learning and Verbal Behavior, 12, 608-617.

Received September 4, 1990 Revision received January 10, 1991 Accepted January 16, 1991

\section{APA Convention "Call for Programs"}

The "Call for Programs" for the 1992 APA annual convention will be included in the October issue of the APA Monitor. The 1992 convention will be held in Washington, DC, from August 14 through August 18. Deadline for submission of program and presentation proposals is December 13, 1991. Additional copies of the "Call" will be available from the APA Convention Office in October. 